\title{
Early Prediction of Functional Recovery after Experimental Stroke: Functional Magnetic Resonance Imaging, Electrophysiology, and Behavioral Testing in Rats
}

\author{
Ralph Weber, ${ }^{1,2 \star}$ Pedro Ramos-Cabrer, ${ }^{1,3 \star}$ Carlos Justicia, ${ }^{1,4}$ Dirk Wiedermann, ${ }^{1}$ Cordula Strecker, ${ }^{1}$ \\ Christiane Sprenger, ${ }^{1}$ and Mathias Hoehn ${ }^{1}$ \\ ${ }^{1}$ In Vivo NMR Laboratory, Max-Planck-Institute for Neurological Research, 50931 Cologne, Germany, ${ }^{2}$ Department of Neurology, University Duisburg- \\ Essen, 45122 Essen, Germany, ${ }^{3}$ Clinical Neurosciences Research Laboratory, Hospital Clínico Universitario, 15706 Santiago de Compostela, Spain, and \\ ${ }^{4}$ Farmacologia i Toxicologia, Institut d'Investigacions Biomèdiques de Barcelona/Consejo Superior de Investigaciones Científicas, Institut d'Investigacions \\ Biomèdiques August Pi i Sunyer, 08036 Barcelona, Spain
}

Therapeutic success of treatment of cerebral diseases must be assessed in terms of functional outcome. In experimental stroke studies, this has been limited to behavioral studies combined with morphological evaluations and single time point functional magnetic resonance imaging ( $\mathrm{fMRI}$ ) measurements but lacking the access to understanding underlying mechanisms for alterations in brain activation. Using a recently developed blood oxygenation level-dependent fMRI protocol to study longitudinal and intraindividual profiles of functional brain activation in the somatosensory system, we have demonstrated activation reemergence in the original representation field as the basic principle of functional recovery from experimental stroke. No plastic reorganization has been observed at any time point during 7 weeks after stroke induction. Applying combined recording of fMRI and somatosensory evoked potentials, we observed a tight coupling of electrical brain activity and hemodynamic response at all times, indicating persistent preservation of neurovascular coupling. Identification of functional brain recovery mechanisms has important implications for the understanding of brain plasticity after cerebral lesions, whereas preservation of neurovascular coupling is important for the clinical translation of fMRI.

Key words: fMRI; neurovascular coupling; functional reorganization; stroke; functional recovery; ischemia

\section{Introduction}

Focal brain ischemia is a major cause of permanent disability. A better understanding of brain recovery and potential reorganization processes is essential to develop new therapeutic strategies after ischemic brain injury and to improve rehabilitation methods.

To date, longitudinal functional magnetic resonance imaging (fMRI) studies in patients with ischemic stroke are scarce because parallel concomitant disease factors make the interpretation in heterogeneous patient pools difficult. fMRI investigations in animals have the major advantage to combine the noninvasive imaging techniques with invasive methods such as direct recording of cellular activity (Logothetis et al., 2001) and histology (Dijkhuizen et al., 2001; Dijkhuizen and Nicolay, 2003) to provide better insight into mechanisms of fMRI signal changes and underlying pathophysiology. To date, studies in rodents subjected to focal cerebral ischemia (Abo et al., 2001; Dijkhuizen et

\footnotetext{
Received July 6, 2007; revised Nov. 7, 2007; accepted Nov. 15, 2007.

This work was supported by grants from the Hertie Foundation (Functional Brain Imaging) and through European Union Project LSHB-CT-2006-037526 (StemStroke).

* R.W. and P.R.-C. contributed equally to this work.

Correspondence should be addressed to Dr. Mathias Hoehn, In Vivo NMR Laboratory, Max-Planck-Institute for Neurological Research, Gleuelerstrasse 50, D-50931 Cologne, Germany. E-mail: mathias@nf.mpg.de.

D0I:10.1523/JNEUROSCI.4147-07.2008

Copyright $\odot 2008$ Society for Neuroscience $\quad$ 0270-6474/08/281022-08\$15.00/0
}

al., 2001, 2003; Sauter et al., 2002) have, however, also not been performed longitudinally because of limitations of anesthesia. The studies performed so far used contrast-enhanced changes of cerebral blood volume (CBV) instead of blood oxygenation leveldependent (BOLD) MR imaging, to visualize brain activation and its recovery. Furthermore, in those reports, measurements of hemodynamic changes had not been combined with direct assessment of electrical activity to investigate mechanisms of neurovascular coupling, thus omitting the chance to systematically assess controversial reports about alterations of neurovascular coupling after focal brain ischemia in humans (Pineiro et al., 2002; Rossini et al., 2004; Krainik et al., 2005).

Recently, we developed a protocol allowing for repetitive independent sessions of noninvasive BOLD fMRI studies of rat forepaw stimulation (Ramos-Cabrer et al., 2005; Weber et al., 2006). Using this approach, we investigated for the first time longitudinal brain reorganization in the somatosensory system of rats subjected to focal ischemic brain injury and mechanisms of coupling between neural activity and hemodynamic response in the acute, the early and late chronic stage after focal brain ischemia. Design of our study was performed to investigate our three major hypotheses. (1) We questioned the validity of lesion volume as the predominant parameter determining clinical outcome and hypothesized that the temporal pattern of functional brain activity is a more reliable factor for the prediction of func- 
tional outcome. (2) We hypothesized that the neurovascular coupling remains preserved in experimental stroke during all phases after stroke induction, thus allowing the conclusion about functional brain activity to be extracted from fMRI results under these pathophysiological conditions. (3) We further hypothesized that recovery of the original representation field is the dominant mechanism of functional recovery in the somatosensory system, as opposed to the assumption of major plastic reorganization as reason for functional improvement.

In our present investigation, we induced stroke in rats and studied the animals for 7 weeks. This stroke-dependent longterm profile included data on morphological lesion evolution as well as information on electrical brain activity using somatosensory evoked potentials (SSEP) together with parallel recording of the hemodynamic response to the stimulus, measured with BOLD fMRI. The study was complemented with behavioral tests analyzing the somatosensory system.

\section{Materials and Methods}

All experiments were performed in accordance with the National Institutes of Health animal protection guidelines and were approved by the local governmental authorities.

Stroke induction. Twenty-two male Wistar rats (330-390 g; HarlanWinkelmann, Borchen, Germany) were used; 19 of these were subjected to $60 \mathrm{~min}$ of transient middle cerebral artery occlusion (MCAO) by the intraluminal thread occlusion method (Longa et al., 1989) under $1 \%$ halothane anesthesia in $65 \% \mathrm{~N}_{2} \mathrm{O} / 35 \% \mathrm{O}_{2}$. The occlusion period and the successful reperfusion of the right MCA were controlled by ipsilateral laser-Doppler flowmetry. Four animals died in the first 24 postoperative hours attributable to massive brain edema.

Anesthesia and physiological monitoring. In full agreement with a recently published experimental protocol for longitudinal fMRI experiments (Weber et al., 2006), animals were sedated with subcutaneous application of medetomidine $\left(0.05 \mathrm{mg} / \mathrm{kg}\right.$ bolus and $0.1 \mathrm{mg} \cdot \mathrm{kg}^{-1} \cdot \mathrm{h}^{-1}$ infusion) during the combined fMRI and electrophysiological experiment. Respiration rate and transcutaneous $\mathrm{pCO}_{2}$ were continuously recorded noninvasively, and body temperature was kept constant by a feedback-controlled heating pad (Ramos-Cabrer et al., 2005). After the experiment, sedation was antagonized with an intraperitoneal injection of atipamezole $(0.1 \mathrm{mg} / \mathrm{kg})$. No animal died in the follow-up period.

MRI and stimulation protocol. Repetitive MRI experiments were conducted on a 7.0 T Biospec animal scanner (Bruker BioSpin, Ettlingen, Germany) during the day cycle of the animals, 1 week before stroke induction and $2 \mathrm{~d}, 1$ week, 2 weeks, 4 weeks, and 7 weeks after MCAO. After positioning the animal's head, quantitative $\mathrm{T}_{2}$ measurements were performed with a multislice spin-echo sequence (Eis et al., 1994) [repetition time (TR)/echo time (TE), 3000/7.5 ms; 16 slices of $1 \mathrm{~mm}$ thickness; 16 echoes] to assess $\mathrm{T}_{2}$ relaxation times and $\mathrm{T}_{2}$ lesion volume.

Functional MRI was achieved using BOLD contrast, starting at least $1 \mathrm{~h}$ after the induction of the sedation. Multislice spin-echo echo-planar images (five slices of $2 \mathrm{~mm}$ thickness), centered at $4.7 \mathrm{~mm}$ caudal to the rhinal fissure, were acquired (TR/TE, 3000/30 ms).

Electrical forepaw stimulation was performed using rectangular pulses $(2 \mathrm{~mA}, 3 \mathrm{~Hz}, 0.3 \mathrm{~ms})$ in a paradigm of five blocks consisting each of $45 \mathrm{~s}$ resting period and $15 \mathrm{~s}$ activation period, ending with an additional $45 \mathrm{~s}$ resting period. BOLD fMRI was conducted alternating at least three times between each hemisphere, and animals were allowed to rest for at least 5 min between stimulation sessions. Statistical parametric activation maps were constructed with the software STIMULATE (Strupp, 1996). The time course of each pixel during forepaw stimulation was examined using a paired Student's $t$ test $(p<0.01)$. Only clusters that included at least four adjacent activated pixels were considered as positive activation areas (Forman et al., 1995).

Electrophysiology. Directly after the fMRI measurement, sedated animals $(n=9)$ were transferred to a Faraday cage, and short-latency SSEPs were recorded bilaterally from the primary somatosensory cortices (3.5 $\mathrm{mm}$ lateral at bregma level) of the intact skull, using subcutaneously inserted steel needle electrodes. Reference and ground electrodes were placed at the nose and neck, respectively. Because of the reference electrode on the nose, independent SSEPs are recorded separately from each hemisphere. Unilateral forepaw stimulation resulted in an SSEP signal in the forelimb area of the primary somatosensory cortex (S1FL) contralateral to the stimulated paw. SSEPs were constructed with the program DasyLab (DATALOG, Mönchengladbach, Germany) by averaging the signal recorded of 100 triggered rectangular pulses $(2 \mathrm{~mA}, 3 \mathrm{~Hz}, 0.3 \mathrm{~ms})$, amplified 1000-fold and bandpass filtered between 5 and $1000 \mathrm{~Hz}$. SSEP recording was repeated at least two times for each hemisphere. SSEP latencies and amplitudes were considered significantly abnormal if they differed from the corresponding mean value of the baseline values of each hemisphere by $>2$ SDs.

Behavioral testing. The adhesive tape removal test was used to evaluate sensory and motor deficits (Schallert et al., 2002). Two rectangular stripes of tape $(18 \times 12 \mathrm{~mm})$ were applied, in random order and with equal pressure, to the saphaneous part of the forepaws. Animals were observed while removing the tapes in their home cages. Latency to contact and to remove the right and left tape were recorded in three trials per session, and the mean value of the three trials was calculated. At least 5 min of rest was allowed between each trial. Healthy animals were trained daily, $3 \mathrm{~d}$ before baseline session, which was performed at the day before MCAO. After MCAO, the sessions were performed the day before the combined MRI-electrophysiology experiments during the day cycle of the animals.

Histology. Animals were killed after the last fMRI-electrophysiology experiment under deep halothane anesthesia by perfusion fixation with PBS and 4\% paraformaldehyde (PFA). Brains were removed and postfixed overnight in $4 \%$ PFA, followed by immersion in a $30 \%$ sucrose solution for $3 \mathrm{~d}$. Coronal $40-\mu \mathrm{m}$-thick sections were cut on a freezing microtome (Leica, Nussloch, Germany) and stored free floating. Conventional hematoxylin-eosin and luxol-cresyl-violet stainings were performed on every 12 th section. Immunohistochemical staining against the neuronal-specific nuclear protein antibody NeuN (mouse anti-NeuN, 1:100; Millipore, Billerica, MA) was performed on adjacent sections. The Vectastain ABC Method (Vector Laboratories, Burlingame, CA) and $3^{\prime}, 3^{\prime}$-diaminobenzidine $/ \mathrm{NiCl}_{2}$ were used for visualization of primary antibody binding. All sections were analyzed and digitally photographed using Leica MZ FL III and Leica DM RB microscopes, both equipped with a CCD camera.

Grouping of animals for data analysis. All animals were treated the same and underwent the same experimental protocol. Only during data evaluation were they retrospectively grouped, based on functional criteria instead of using purely morphological lesion volume determination. The first criterion was successful induction of ischemic lesion, observed on $\mathrm{T}_{2}$-weighted $\mathrm{MR}$ images, $24 \mathrm{~h}$ after stroke induction. Then, animals were grouped depending on their long-term capacity for functional restoration. This led to the following classification at the end of the survival period for the whole group. Healthy animals served as control group (group $\mathrm{C} ; n=3$ ). Animals with a clear MCAO-induced ischemic lesion but without detectable functional deficits were collected in group 1 ( $n=$ 4). Primary occurrence of a functional deficit in all three investigative criteria (fMRI, SSEP, and behavior) formed groups 2 and 3. Group $2(n=$ 6) consisted of animals with a primary, but only transient functional deficit (observable on all three investigative criteria; compare with above) followed by partial recovery of the studied functions. Group 3 $(n=5)$ included animals with an unchanged, persistent functional deficit over the whole observation period (7 weeks).

Statistical analysis. All values are expressed as mean \pm SEM, unless stated otherwise. Because of sample sizes, statistical comparisons of $\mathrm{T}_{2}$ lesion volume, BOLD percentage change in the left and right primary somatosensory cortex, and behavioral testing at each time point were performed using Kruskal-Wallis ANOVA and exact testing, followed by the Mann-Whitney $U$ test when appropriate.

The statistical comparison of SSEP amplitudes between the left and right primary somatosensory cortex in healthy animals at baseline was performed using a paired Student's $t$ test. SSEP latencies and amplitudes were considered abnormal if they differed from the corresponding mean value of the baseline values of each hemisphere by $>2$ SDs. 
For correlation analysis between BOLD percentage change and SSEP amplitude and between $\mathrm{T}_{2}$ lesion volume and histologically determined infarct size, the Spearman's rank order correlation test was used. A $p$ value of $<0.05$ was considered significant.

\section{Results}

Ischemic lesion induction by MCAO Invariably, $60 \mathrm{~min}$ occlusion of the right MCA led to a lesion area in the acute phase that always encompassed the striatal territory and often extended into the cortex. Despite the standardized, well established and tightly controlled model, there was distinct interindividual variability in lesion size (see Figs. 1, 5).

Temporal profile of functional brain activation patterns after focal brain ischemia

fMRI

Alternating unilateral electrical stimulation of the right and the left forepaw in healthy Wistar rats resulted in a significant and reproducible BOLD response in the forelimb area of the contralateral primary somatosensory cortex [forelimb area, S1FL (according to the rat brain atlas of Paxinos and Watson, 1998)] in all animals.

Transient occlusion of the right middle cerebral artery for $60 \mathrm{~min}$ did not alter the activation patterns in the S1FL of the left, unaffected hemisphere both in the subacute (day 2) and chronic (weeks 1-7) stages for all study groups (Figs. 1, 2a) but resulted in three different activation patterns in the right, affected hemisphere (Figs. 1, 2b). In the first group of animals

Figure 1. Combined structural brain imaging, functional brain imaging, and SSEP recording after focal ischemic stroke. $\mathrm{T}_{2}$ maps (first column), BOLD spin-echo echo-planar images (SE-EPI) during right (second column) and left (third column) forepaw stimulation, and the corresponding SSEP signals from S1FL (fourth column; left hemispheric SSEP signal in blue; right hemispheric SSEP signal in red) from representative animals with no loss (group 1), transient loss (group 2), and permanent loss (group 3) of BOLD activation in the right primary somatosensory cortex. BOLD activation and normal SSEP signals can be observed in both primary somatosensory cortices in all animals during baseline measurements $(\boldsymbol{B})$, i.e., before transient MCAO. No loss of BOLD activation and normal SSEP signals are found in animals with subcortical infarction (group 1) at all time points during the 7 week observation period. In animals with a transient loss of BOLD activation in the right S1FL area (group 2), the right SSEP signal is delayed and diminished $2 \mathrm{~d}$ after MCAO (D2) and delayed 1 week after MCAO (W1). In parallel with reemergence of $B O L D$ activation 2 weeks after $M C A O$ (W2), a normal SSEP signal is observed again. In animals with permanent loss of BOLD activation in the right S1FL area (group 3), no restoration of the SSEP signal is observed over time. Note that the first spike at time point 0 represents the electrical stimulus in the SSEP recording.

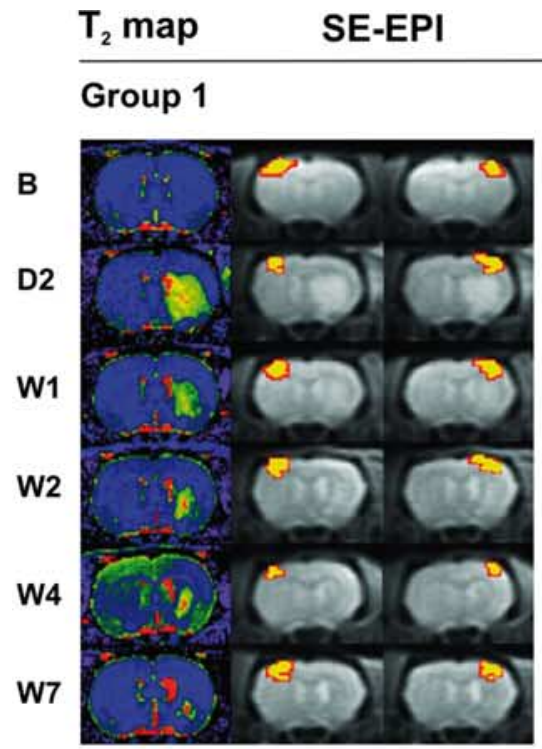

Group 2

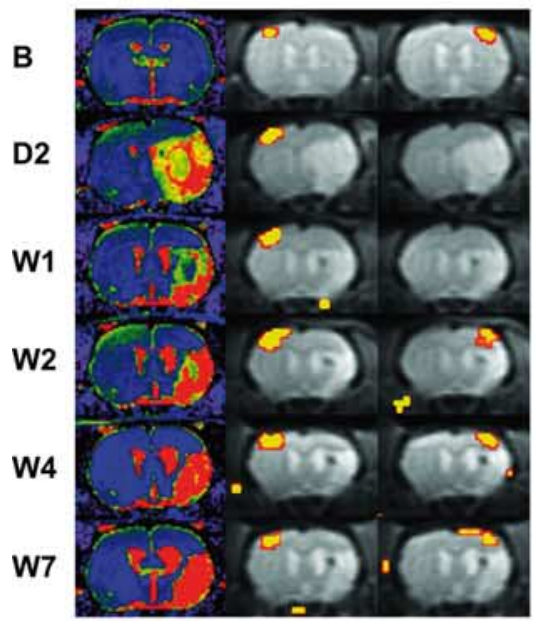

\section{Group 3}

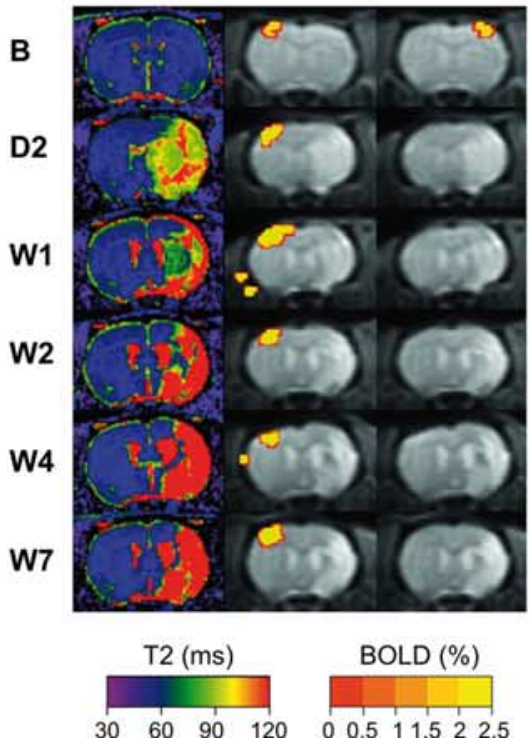

SSEP

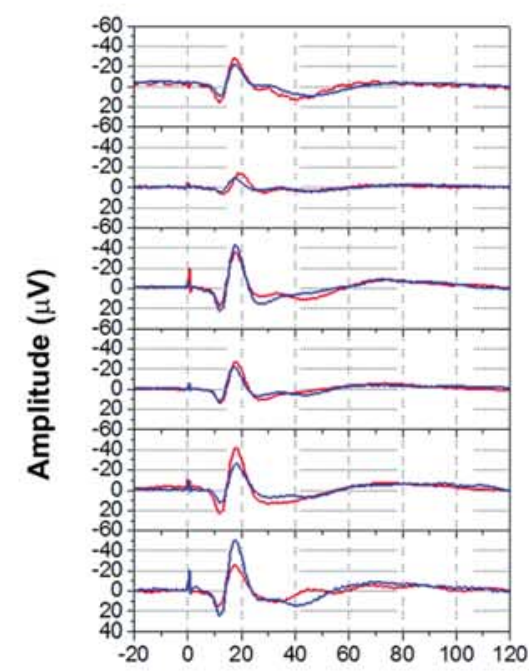

Time (ms)
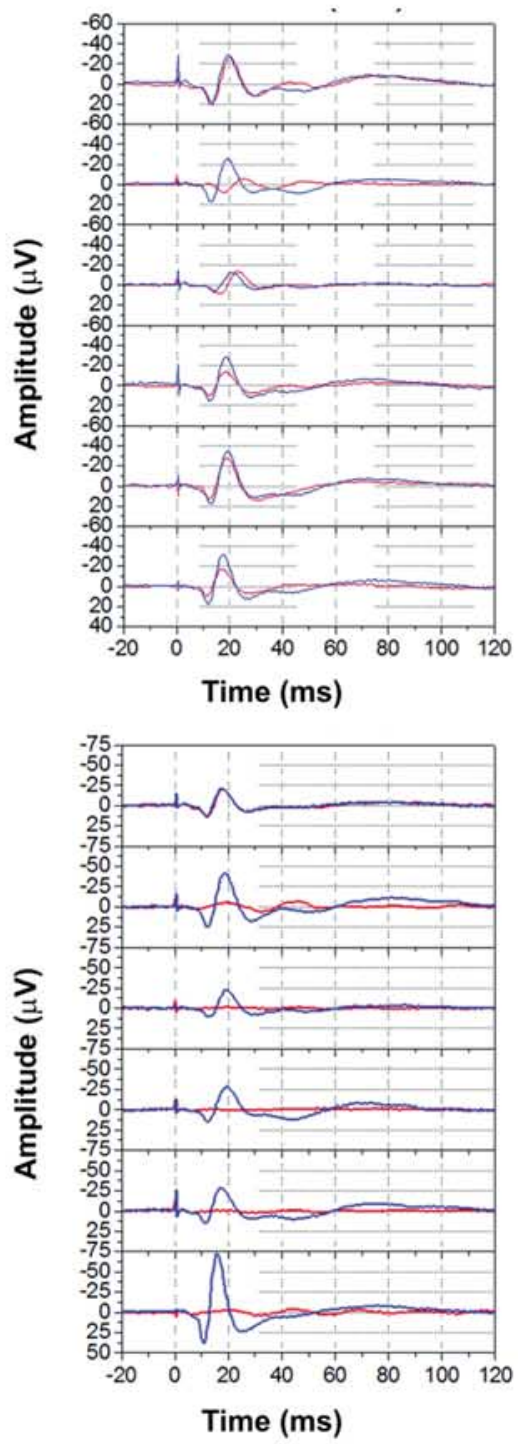
a

Left primary somatosensory cortex
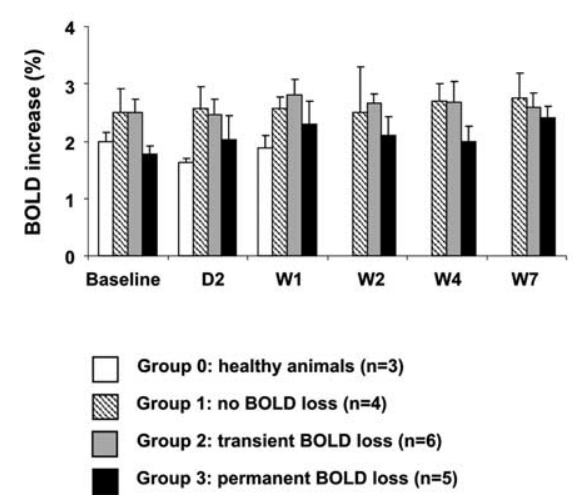

b Right primary somatosensory cortex

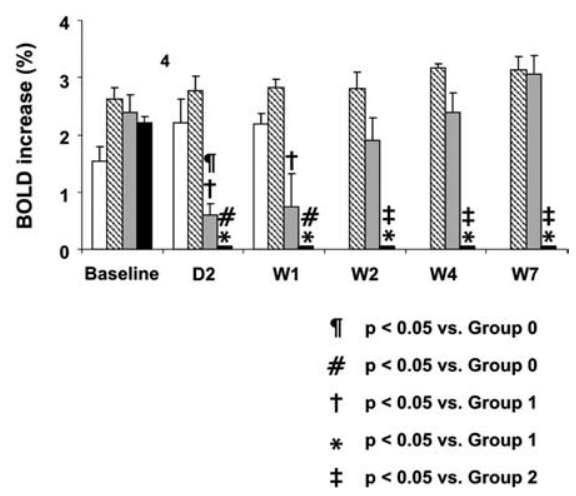

Figure 2. BOLD percentage change in the left $(\boldsymbol{a})$ and right $(\boldsymbol{b})$ primary somatosensory cortex during electrical forepaw stimulation before (baseline) and $2 \mathrm{~d}$ (D2), 1 week (W1), 2 weeks (W2), 4 weeks (W4), and 7 weeks (W7) after MCA0. Healthy animals were only studied at the first three time points. No positive BOLD percentage change was observed in animals with permanent BOLD loss after MCAO in the right primary somatosensory cortex. Data represent mean \pm SEM.

(group $1 ; n=4$ ), a significant BOLD response was invariably observed in the right S1FL at all follow-up fMRI measurements, indistinguishable from the control group (Fig. $2 b$ ). The animals of group 1 either developed selective neuronal death $(n=2)$ or infarction $(n=2)$ of the right striatum.

In the second group of animals (group $2 ; n=6$ ), three rats developed an infarction of the right striatum, whereas three animals had a combined infarction of the right striatum and parietal cortex. Stimulation of the left forepaw in this group resulted in either a transient decrease or transient loss of the BOLD response in the subacute and early chronic stage after MCAO. At day 2 after stroke induction, this reduction of BOLD response was significant relative to the control group $(p=0.012)$ and relative to group 1 (no loss of BOLD activity; $p=0.001$ ), whereas during the following weeks a clear trend toward normalization was observed (Fig. 2b). Complete reemergence of normal activation in the right S1FL occurred between 1 and 4 weeks after MCAO in all animals. Functional reorganization in remote brain areas was not observed at any time point.

In the third group (group 3; $n=5$ ), all animals developed a combined infarction of the right striatum and parietal cortex. Stimulation of the left forepaw after transient MCAO did not result in a statistically significant BOLD response in the right S1FL or any other brain area during the following 7 weeks in this group (Figs. 1, 2b).

\section{Electrical activity and hemodynamic response after stroke}

Combined electrophysiological recording of short-latency SSEPs and BOLD fMRI were used to investigate the neuronal activity and hemodynamic response before and after stroke induction (Figs. 1, 2). This combined dataset over time was exploited to investigate potential disturbances of the neurovascular coupling during stimulation and after stroke.

SSEP recording of the primary somatosensory cortex in healthy animals revealed a typical waveform with a first positive peak (P1) followed by a first negative (N1) and a second positive peak, similar to the SSEP signals recorded from intracranially or transcranially implanted electrodes (Sakatani et al., 1990; Ogawa et al., 2000; Gsell et al., 2006). No significant interhemispheric differences for latency of $\mathrm{P} 1$ (mean $\pm \mathrm{SD} ; \mathrm{P}_{\text {left, }}, 12.4 \pm 0.6 \mathrm{~ms}$; $\left.\mathrm{P} 1_{\text {right }}, 12.3 \pm 0.5 \mathrm{~ms} ; p=0.62\right)$, for $\mathrm{N} 1$ latency $\left(\mathrm{N}_{\text {left }}, 18.3 \pm 0.8\right.$ $\mathrm{ms} ; \mathrm{N} 1_{\text {right }}, 18.2 \pm 1.3 \mathrm{~ms} ; p=0.71$ ), or for the amplitude between these two peaks (amplitude left, $31 \pm 9.3 \mu \mathrm{V}$; amplitude $_{\text {right, }}$,
$33.8 \pm 9.7 \mu \mathrm{V} ; p=0.21)$ were observed. Latencies and amplitudes remained unchanged at all time points after MCAO for the S1FL recording site of the left, unaffected hemisphere in all animals, regardless of the extent of brain ischemia.

After transient MCAO, SSEP recording in animals with preserved BOLD response in the right S1FL (groups C and 1) showed changes in neither the peak latencies nor the amplitudes at both S1FL recording sites. SSEP recording in animals with transient loss of BOLD activation (group 2) revealed a significant amplitude reduction and/or prolonged latencies for $\mathrm{P} 1$ and N1 when no significant BOLD response was present in the right S1FL (Table 1). Reemergence of a significant BOLD response was always connected with the restoration of normal SSEP latencies and amplitudes.

Animals presenting a permanent loss of the BOLD response in the right S1FL (group 3) had no recordable SSEP signal or a marked abnormal amplitude reduction over the right S1FL. No restoration of the SSEP signal occurred during the complete study period in these animals (Table 1). A SSEP signal in the left (ipsilateral) S1FL during electrical stimulation of the left, affected forepaw (that would have indicated interhemispheric reorganization) was not observed at any time point.

Correlation analysis between BOLD percentage change and SSEP amplitude in the right S1FL during left forepaw stimulation showed a correlation coefficient of $0.884(p<0.0001)$ for all time points (Fig. 3). A significantly detectable, but very weak BOLD percentage change $(0.3 \%)$ was only detected in one animal with an SSEP amplitude $<14 \mu \mathrm{V}$. SSEP amplitudes $>50 \mu \mathrm{V}$ did not result in a higher BOLD percentage change than $3.8 \%$.

\section{Behavioral testing for functional outcome}

To study functional outcome, behavioral tests were always performed on the day before the combined fMRI-electrophysiology experiments (Fig. 4). To assess the somatosensory pathway, we focused on the latency-to-contact of both forepaws with the adhesive tape removal test (Schallert et al., 2002).

All animals during baseline testing and animals developing no ischemic injury (group C) demonstrated no significant differences in their latency to contact the tape between the left and the right paw. Whereas the latency to contact the tape on the unimpaired right forepaw was not significantly affected by the transient MCAO at any time point (Fig. 4a), the latency to contact the tape on the impaired left forepaw was prolonged in all animals presenting an infarction of the right hemisphere (Fig. 4b), yet differences in both the degree of impairment and recovery were obvious between the three different groups.

Animals with preservation of their BOLD response (group 1) showed only a minor and nonsignificant prolongation of the latency to contact the left tape $2 \mathrm{~d}$ after MCAO, which remained prolonged at the same level at all follow-up time points.

Animals with a BOLD reemergence (group 2) presented a significant prolongation to contact the tape on the left, affected paw when compared with healthy animals 1 week after MCAO $(p=0.025)$ (Fig. 4b). These animals constantly improved during edema resorption toward week 7 , although not reaching preischemic latencies or amplitudes of animals with no loss of BOLD response or healthy animals. 
Table 1. SSEP amplitudes ( $\mu \mathrm{V})$ and first positive peak latency $(\mathrm{P} 1 ; \mathrm{ms})$ in the right (ischemic) hemisphere during stimulation of the contralateral forepaw at preischemic (Baseline) and postischemic time points

\begin{tabular}{|c|c|c|c|c|c|c|c|c|c|c|c|c|c|}
\hline \multicolumn{2}{|c|}{ Time point } & \multicolumn{2}{|l|}{ Baseline } & \multicolumn{2}{|l|}{ Day 2} & \multicolumn{2}{|l|}{ Week 1} & \multicolumn{2}{|l|}{ Week 2} & \multicolumn{2}{|l|}{ Week 4} & \multicolumn{2}{|l|}{ Week 7} \\
\hline Animal & Group & SSEP amplitude & P1 latency & SSEP amplitude & P1 latency & SSEP amplitude & P1 latency & SSEP amplitude & P1 latency & SSEP amplitude & P1 latency & SSEP amplitude & P1 latency \\
\hline 22058 & 1 & 44 & 13.0 & 47 & 13.0 & 55 & 12.5 & n.d. & n.d. & 55 & 12.5 & 49 & 12.0 \\
\hline 22059 & 1 & 33 & 12.0 & 30 & 12.5 & 32 & 13.0 & n.d. & n.d. & 35 & 12.0 & 29 & 12.0 \\
\hline 22060 & 1 & 45 & 12.0 & 22 & 13.5 & 66 & 12.0 & 41 & 12.0 & 65 & 12.0 & 41 & 11.5 \\
\hline 22080 & 1 & 29 & 12.0 & 15 & 13.5 & 29 & 11.5 & 41 & 12.0 & 64 & 12.0 & 74 & 12.0 \\
\hline 22067 & 2 & 45 & 13.0 & $8^{a}$ & $14^{a}$ & $14^{a}$ & 11.0 & $8^{a}$ & 11.5 & 25 & 11.5 & 32 & 12.0 \\
\hline 22081 & 2 & 29 & 12.5 & 22 & $16.5^{a}$ & $14^{a}$ & $17.5^{a}$ & 24 & 12.0 & 40 & 12.0 & 49 & 11.5 \\
\hline 22068 & 3 & 31 & 12.0 & $0^{b}$ & $0^{b}$ & $0^{b}$ & $0^{b}$ & $0^{b}$ & $0^{b}$ & $0^{b}$ & $0^{b}$ & $7^{a}$ & 11.5 \\
\hline 22069 & 3 & 15 & 12.0 & $0^{b}$ & $0^{b}$ & $0^{b}$ & $0^{b}$ & $0^{b}$ & $0^{b}$ & $0^{b}$ & $0^{b}$ & $0^{b}$ & $0^{b}$ \\
\hline 22070 & 3 & 34 & 12.0 & $0^{b}$ & $0^{b}$ & $0^{b}$ & $0^{b}$ & $0^{b}$ & $0^{b}$ & $0^{b}$ & $0^{b}$ & $0^{b}$ & $0^{b}$ \\
\hline
\end{tabular}

${ }^{a}$ Abnormal amplitude reduction and abnormal prolongation of P1 latency ( $\geq 2$ SDs from baseline value).

${ }^{b}$ No SSEP signal was recorded on these post-ischemic time points.

n.d., SSEP signal has not been measured in these two animals 2 weeks after MCAO.

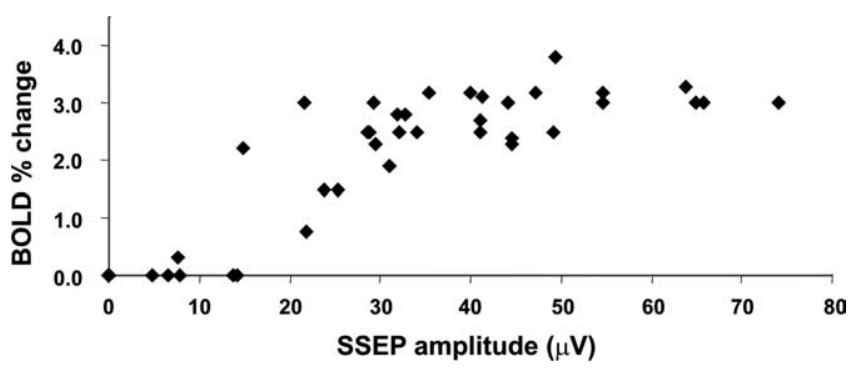

Figure 3. Correlation between BOLD percentage change and SSEP amplitude in the right affected primary somatosensory cortex (S1FL) during electrical forepaw stimulation of the left forepaw. Data are compiled for all animals and at all time points (Spearman's $r=0.884 ; p<$ $0.0001)$. Note the symbol at the coordinate system zero represents 13 measurements without BOLD and SSEP signals.

Animals with permanent loss of their BOLD response (group 3 ) showed a significant prolongation to contact the tape on the left paw when compared with healthy animals, both $2 \mathrm{~d}$ and at 1 week after MCAO ( $p=0.024$ and $p=0.025$, respectively). These animals also improved during edema resorption but slightly deteriorated again from week 2 to week 7 after MCAO (Fig. 4b). This group performed worst during the chronic observation period.

\section{Temporal profile of morphological lesion after focal brain ischemia}

Analysis of quantitative $\mathrm{T}_{2}$ maps showed a $\mathrm{T}_{2}$ relaxation time of $55.4 \pm 1.0$ and $51.3 \pm 0.4 \mathrm{~ms}$ in normal cortex and striatum, respectively. This value remained indistinguishable between preischemic values and postischemic values of the healthy hemisphere at all times. Two days after stroke induction, the ischemic lesion was clearly visible as a hyperintense area on $\mathrm{T}_{2}$-weighted $\mathrm{MR}$ images. The ischemic $\mathrm{T}_{2}$ value increased to $76.6 \pm 1.6,88.3 \pm$ 3.9 , and $94.5 \pm 1.1 \mathrm{~ms}$ in groups 1, 2, and 3, respectively. During the following 7 weeks, $\mathrm{T}_{2}$ values of group 1 remained essentially stable at this level (Fig. 4$)\left(\mathrm{T}_{2}\right.$ at 7 weeks, $\left.75.6 \pm 12.7 \mathrm{~ms}\right)$. In groups 2 and 3, conversely, a continuous increase in $\mathrm{T}_{2}$ was observed. In group 2, this increase resulted in an ischemic $\mathrm{T}_{2}$ value of $126.9 \pm 11.7 \mathrm{~ms}$ after 7 weeks, whereas the corresponding increase in group 3 was very pronounced $\left(\mathrm{T}_{2}\right.$ at 7 weeks, $168.1 \pm$ $14.5 \mathrm{~ms})$.

Correspondingly, the evolution of the apparent $\mathrm{T}_{2}$ lesion volume differed. In group 1 , the $\mathrm{T}_{2}$-defined lesion volume was highest at $2 \mathrm{~d}$ after stroke induction $\left(63.3 \pm 15.2 \mathrm{~mm}^{3}\right)$ and then continuously reduced to reach $8.8 \pm 4.2 \mathrm{~mm}^{3}$ after 7 weeks. In group 2, $\mathrm{T}_{2}$ lesion volume was $124.1 \pm 28.5 \mathrm{~mm}^{3}$ at day 2 , followed by a continuous reduction to $55.2 \pm 23.3 \mathrm{~mm}^{3}$ after 7 weeks. In group 3, $\mathrm{T}_{2}$ lesion volume was $198.2 \pm 12 \mathrm{~mm}^{3}$ after $2 \mathrm{~d}$ and slowly decreased to $143.0 \pm 15.3 \mathrm{~mm}^{3}$ after 7 weeks (Fig. 5).

\section{Correlation between $\mathrm{T}_{2}$-based lesion and functional deficit}

To assess the interpretation of $\mathrm{T}_{2}$-weighted MRI hyperintensity as representing the true lesion size, we determined the lesion area on histological sections through the center of the lesion. This lesion area was compared with the area of elevated $T_{2}$ value on the $\mathrm{T}_{2}$ maps of the last session, at 7 weeks, directly before killing the animals for histological analysis. Small lesions will not necessarily lead to pannecrosis but only to selective neuronal death (group 1 ), which can lead to normalization of $\mathrm{T}_{1}$ and $\mathrm{T}_{2}$ relaxation times during the chronic stroke period (Wegener et al., 2006).

The correlation analysis between $\mathrm{T}_{2}$ lesion volume and histologically determined infarction size was therefore limited to the data of groups 2 and 3 in which pannecrosis was always detectable on histological sections. This resulted in a good linear correlation between $\mathrm{T}_{2}$-based lesion size and histological lesion determination with a correlation coefficient $r=0.85$, indicating that the $\mathrm{T}_{2}$-visible lesion does reflect the necrotic tissue area during the chronic phase of the lesion evolution in these two groups.

We further analyzed whether $\mathrm{T}_{2}$-based lesions allow a morphological prediction of functional deficit or outcome. However, although $\mathrm{T}_{2}$ lesion volume was significantly different in the chronic phase between animals with a transient and permanent BOLD loss (a consequence of the mixture of pure subcortical infarcts and subcortico-cortical infarcts in the group with transient BOLD loss), when investigating the BOLD pattern in individual animals, it became obvious that no connection exists between lesion size and functional brain activation potential. As a matter of fact, extreme cases were observed in which functional brain activation still persisted despite very large $T_{2}$ lesions, whereas in other cases smaller lesions led to a permanent inhibition of functional activation (Fig. 6).

\section{Discussion}

\section{Intraindividual longitudinal fMRI monitoring during} lesion evolution

To our knowledge, this is the first animal fMRI study permitting an intraindividual temporal profile. Previous fMRI studies used $\alpha$-chloralose in which functional-metabolic coupling is well preserved (Ueki et al., 1988). However, severe side effects of $\alpha$-chloralose (Silverman and Muir, 1993; Hedenquist and Hellebrekers, 2003) make it unsuitable for longitudinal fMRI studies, 

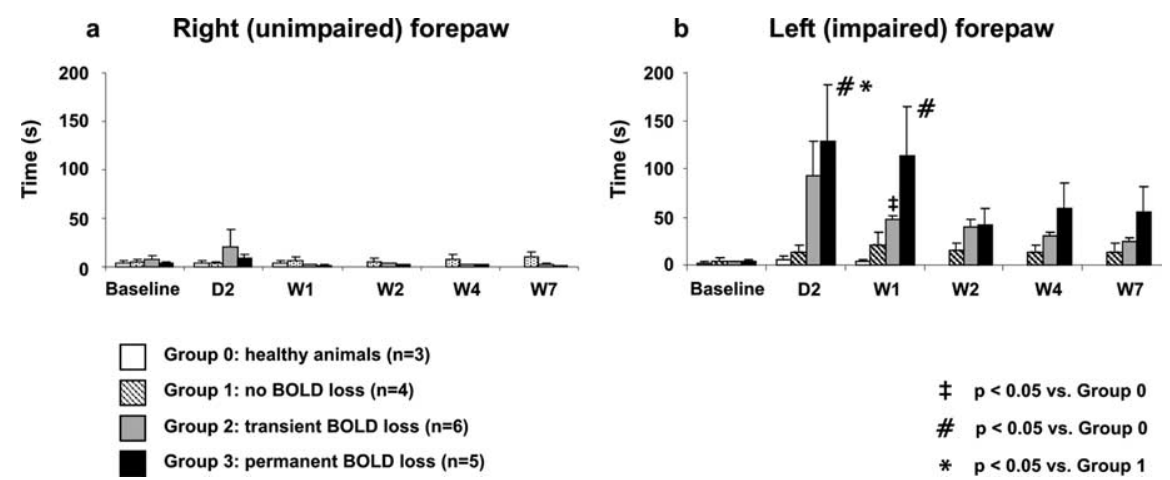

Figure 4. Behavioral testing after stroke. Time to first contact of the right, unimpaired forepaw $(\boldsymbol{a})$ and left, impaired forepaw (b) in the adhesive tape removal test before (baseline) and $2 \mathrm{~d}$ (D2), 1 week (W1), 2 weeks (W2), 4 weeks (W4), and 7 weeks (W7) after MCA0. Healthy animals were tested only at the first three time points. Data represent mean \pm SEM. but will not permit reliable prediction of long-term functional outcome on an individual level. Spontaneous recovery of functional brain activation exists in cases of almost hemispheric ischemic involvement, whereas functional deficit may persist in equivalent or even smaller lesions (Fig. 6).

\section{Functional outcome is reflected in} BOLD fMRI activation pattern and confirms reemergence of activation as basic principle of brain reorganization Our intraindividual time profile studies showed a stable pattern: those animals with a chance of recovery gained significant BOLD activation in the primary somatosensory cortex 1-4 weeks after stroke at the latest, independent of lesion size. Animals that did not recover within the first 4 weeks did not show significant BOLD activation over the next 6 months (unpublished data; Ramos-Cabrer and Justicia) and presented the worst functional outcome in behavioral testing after edema resorption. The finding that reemergence of activation in the primary somatosensory cortex is the basic principle of brain reorganization in the somatosensory system is in agreement with recent functional imaging reports in both rats (Dijkhuizen et al., 2001, 2003) and humans (Wikstrom et al., 2000; Carey et al., 2002; Oliviero et al., 2004).

In contrast to previous reports in rats (Abo et al., 2001; Dijkhuizen et al., 2001, 2003), no signs of brain plasticity in both the affected and unaffected hemisphere were observed here. Even in severely affected animals with no restoration of electrical and BOLD activation in the primary somatosensory cortex, no BOLD activation was seen in other brain regions. Dijkhuizen and colleagues reported activation in the unaffected cortex $3 \mathrm{~d}$ after stroke induction. This trans-hemispheric shift resolved when activation was restored in the primary somatosensory cortex of the affected hemisphere after $14 \mathrm{~d}$ (Dijkhuizen et al., 2001, 2003). In a study in which the primary somatosensory cortex was directly lesioned (Abo et al., 2001), activation of the sensorimotor cortex of the unaffected hemisphere and activation lateral to the lesion was observed 3 weeks after stroke induction, at a time when all animals showed complete functional recovery. Whereas the aforementioned authors measured changes of CBV after injection of an intravascular contrast agent, we used the BOLD fMRI method, because it is noninvasive, can be easily repeated in a longitudinal study, and is used in the vast majority of human $\mathrm{fMRI}$ studies.

The BOLD fMRI signal depends on blood volume, blood flow, and oxygenation (Heeger and Ress, 2002; Logothetis and Wandell, 2004). It was reported that activation-induced CBV changes are more sensitive compared with BOLD signal changes in rats subjected to transient MCAO (Kim et al., 2005), but the interpretation of CBV-based fMRI is also critically discussed in the literature (Smirnakis et al., 2007). The lower sensitivity of BOLD fMRI could account for the lack of detection of brain plasticity in our study. Recently, an electrophysiological threshold for the coupling of neuronal activity and cerebral blood flow in the somatosensory cortex of healthy rats was reported (Norup Nielsen and Lauritzen, 2001). Figure 3 shows lack of BOLD for SSEPs smaller than $14 \mu \mathrm{V}$, but, because of our experimental setup, SSEP amplitudes below $10 \mu \mathrm{V}$ are not significantly above the noise level. Thus, we may not have been able to detect electrical activation during forepaw stimulation below this threshold value of 14
Lack of correlation between lesion size and functional deficit Past experimental and clinical studies assessed ischemic lesion severity based on lesion volume as the determining parameter (Palmer et al., 2001). Concerning metabolic and hemodynamic alterations and recovery, this approach may be valid because it characterizes the tissue volume affected by the ischemic condition. However, as shown here, lesion volume alone will at best provide a statistically relevant trend across group comparisons 


\section{BOLD}

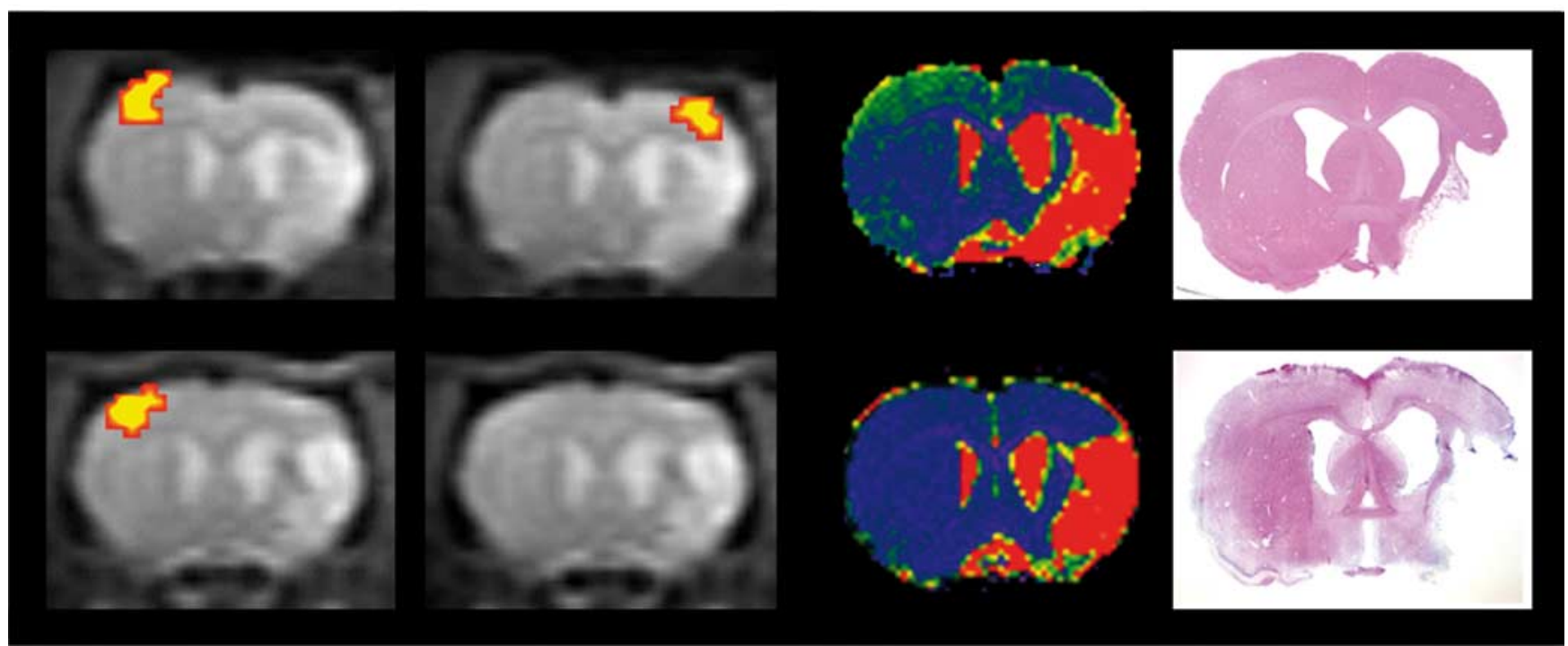

Figure 6. Comparison of lesion size and functional deficit condition at 7 weeks after MCAO. The first two images show the BOLD response after stimulation of both forepaws. The $\mathrm{T}_{2}$ image demarcates the $\mathrm{T}_{2}$ lesion size, in good agreement with the hematoxylin- eosin (HE) staining. The top row shows an animal with a rather large cortico-subcortical lesion but full restitution of BOLD fMRI signal in the ischemic hemisphere at 7 weeks. The bottom row presents an animal with a somewhat smaller lesion but with persistent functional deficit at 7 weeks (i.e., no BOLD recovery of the ischemic hemisphere).

$\mu \mathrm{V}$ in both the primary somatosensory cortex and remote brain areas resulting in a significant BOLD response in our study.

Furthermore, differences in the MCAO stroke models ( 90 or 120 min MCAO vs 60 min MCAO) may have lead to a gradual difference in lesion severity and induction of transient brain reorganization in more affected animals. Because our new anesthesia protocol for fMRI has only been compared with $\alpha$-chloralose in healthy animals so far (Ramos-Cabrer et al., 2005; Weber et al., 2006), we cannot rule the possibility that $\alpha 2$-noradrenergic agonists such as medetomidine could alter or even suppress brain plasticity after stroke (Feeney et al., 2004).

\section{Preservation of neurovascular coupling after stroke}

By longitudinally combining noninvasive functional imaging and electrophysiological recording of SSEPs, the underlying causes of activation loss and restoration are better characterized. Activation reemergence in the somatosensory cortex was only registered by BOLD fMRI, when electrical activity was restored 1-4 weeks after induction of stroke. During the period of loss of electrical activity to the somatosensory stimulus, brain edema formation, followed by gradual reabsorption (Rossini et al., 2003), and impaired neurotransmission (Aoyagi et al., 1998) may be regarded as responsible for the transient dysfunction of the electrical activity. Furthermore, activation restoration was only observed when the primary somatosensory cortex was not directly affected by the ischemia.

The correlation of fMRI results with electrophysiological data are important. Although BOLD fMRI is proportional to the SSEPs in anesthetized rats (Brinker et al., 1999) and awake humans (Arthurs et al., 2000) under normal physiological conditions, this coupling between electrical activity and hemodynamic response may be disturbed or even inhibited during pathophysiological conditions. After resuscitation after cardiac arrest in the rat, the cerebral vasculature is transiently paralyzed, mainly because of extracellular acidosis acting as a potent vasodilator (Waltz, 1969; Hossmann, 1997). During the loss of vascular re- activity, electrical activity to somatosensory stimulation was recovering already, leading to a transient neurovascular decoupling (Schmitz et al., 1998). Therefore, a parallel recording of SSEP and BOLD fMRI during the chronic poststroke phase permits assessment of the long-term characterization of neurovascular coupling. Here, we show that, during the first 7 weeks after $1 \mathrm{~h}$ of MCA occlusion, the neurovascular coupling remains preserved at all times.

Kim et al. (2005) discussed stroke-related alterations in hemodynamic coupling, which were controversially reported in human BOLD fMRI studies of sensorimotor stroke recovery (Pineiro et al., 2002; Rossini et al., 2004; Krainik et al., 2005). Two of these human studies reported a decreased BOLD signal in both affected and unaffected sensorimotor cortices of stroke patients compared with age-matched controls (Pineiro et al., 2002; Rossini et al., 2004). In contrast to these observations, we did not observe uncoupling of neuronal activity and BOLD response in both hemispheres but a coupled alteration of both neuronal activity and hemodynamic response. A distorted or absent neuronal activity resulted in decreased or absent hemodynamic response. During restoration of normal neuronal activity, restoration of normal hemodynamic response occurred. A major difference between human and animal stroke fMRI studies are concomitant factors in human stroke patients, which can influence the neurovascular coupling. In both human studies, all reported patients had at least one cardiovascular risk factor, such as arterial hypertension, smoking, and hypercholesterolaemia, and were treated with various medications. All these factors can have marked influence on the vascular reactivity and therefore may induce uncoupling of neuronal activity and hemodynamic response in both the affected and unaffected hemisphere of stroke patients. Under controlled experimental stroke situations in rats, these confounding factors can safely be excluded and are not influencing the hemodynamic coupling.

In summary, our studies reveal the following aspects on functional brain activation in relation to experimental stroke in rats using BOLD fMRI: our data confirm previous studies that acti- 
vation reemergence in the original cortical representation field is the basic principle of functional recovery after stroke in the somatosensory system. Our findings of continuing preservation of neurovascular coupling in both the early and late chronic phase after experimental stroke have important implications for the clinical translation: the coupling preservation indicates that BOLD fMRI may safely be applied as a surrogate marker for functional loss and its restoration in such animal studies.

\section{References}

Abo M, Chen Z, Lai LJ, Reese T, Bjelke B (2001) Functional recovery after brain lesion-contralateral neuromodulation: an fMRI study. NeuroReport 12:1543-1547.

Aoyagi A, Saito H, Abe K, Nishiyama N (1998) Early impairment and late recovery of synaptic transmission in the rat dentate gyrus following transient forebrain ischemia in vivo. Brain Res 799:130-137.

Arthurs OJ, Williams EJ, Carpenter TA, Pickard JD, Boniface SJ (2000) Linear coupling between functional magnetic resonance imaging and evoked potential amplitude in human somatosensory cortex. Neuroscience 101:803-806.

Brinker G, Bock C, Busch E, Krep H, Hossmann KA, Hoehn-Berlage M (1999) Simultaneous recording of evoked potentials and $\mathrm{T} 2^{*}$-weighted MR images during somatosensory stimulation of rat. Magn Reson Med 41:469-473.

Carey LM, Abbott DF, Puce A, Jackson GD, Syngeniotis A, Donnan GA (2002) Reemergence of activation with poststroke somatosensory recovery: a serial fMRI case study. Neurology 59:749-752.

Dijkhuizen RM, Nicolay K (2003) Magnetic resonance imaging in experimental models of brain disorders. J Cereb Blood Flow Metab 23:1383-1402.

Dijkhuizen RM, Ren J, Mandeville JB, Wu O, Ozdag FM, Moskowitz MA, Rosen BR, Finklestein SP (2001) Functional magnetic resonance imaging of reorganization in rat brain after stroke. Proc Natl Acad Sci USA 98:12766-12771.

Dijkhuizen RM, Singhal AB, Mandeville JB, Wu O, Halpern EF, Finklestein SP, Rosen BR, Lo EH (2003) Correlation between brain reorganization, ischemic damage, and neurologic status after transient focal cerebral ischemia in rats: a functional magnetic resonance imaging study. J Neurosci 23:510-517.

Eis M, Els T, Hoehn-Berlage M, Hossmann KA (1994) Quantitative diffusion MR imaging of cerebral tumor and edema. Acta Neurochir Suppl (Wien) 60:344-346.

Feeney DM, De Smet AM, Rai S (2004) Noradrenergic modulation of hemiplegia: facilitation and maintenance of recovery. Restor Neurol Neurosci 22:175-190.

Forman SD, Cohen JD, Fitzgerald M, Eddy WF, Mintun MA, Noll DC (1995) Improved assessment of significant activation in functional magnetic resonance imaging (fMRI): use of a cluster-size threshold. Magn Reson Med 33:636-647.

Gsell W, Burke M, Wiedermann D, Bonvento G, Silva AC, Dauphin F, Bührle C, Hoehn M, Schwindt W (2006) Differential effects of NMDA and AMPA glutamate receptors on functional magnetic resonance imaging signals and evoked neuronal activity during forepaw stimulation of the rat. J Neurosci 26:8409-8416.

Hedenquist P, Hellebrekers LJ (2003) Laboratory animal analgesia, anesthesia and euthanasia. In: Handbook of laboratory animal science (Hau J, Van Hoosier GLJ, eds), pp 413-455. Boca Raton, FL: CRC.

Heeger DJ, Ress D (2002) What does fMRI tell us about neuronal activity? Nat Rev Neurosci 3:142-151.

Hossmann KA (1997) Reperfusion of the brain after global ischemia-hemodynamic disturbances. Shock 8:95-101.

Kim YR, Huang IJ, Lee SR, Tejima E, Mandeville JB, van Meer MP, Dai G, Choi YW, Dijkhuizen RM, Lo EH, Rosen BR (2005) Measurements of $\mathrm{BOLD} / \mathrm{CBV}$ ratio show altered $\mathrm{fMRI}$ hemodynamics during stroke recovery in rats. J Cereb Blood Flow Metab 25:820-829.

Krainik A, Hund-Georgiadis M, Zysset S, von Cramon DY (2005) Regional impairment of cerebrovascular reactivity and BOLD signal in adults after stroke. Stroke 36:1146-1152.
Logothetis NK, Wandell BA (2004) Interpreting the BOLD signal. Annu Rev Physiol 66:735-769.

Logothetis NK, Pauls J, Augath M, Trinath T, Oeltermann A (2001) Neurophysiological investigation of the basis of the fMRI signal. Nature 412:150-157

Longa EZ, Weinstein PR, Carlson S, Cummins R (1989) Reversible middle cerebral artery occlusion without craniectomy in rats. Stroke 20:84-91.

Norup Nielsen A, Lauritzen M (2001) Coupling and uncoupling of activitydependent increases of neuronal activity and blood flow in rat somatosensory cortex. J Physiol (Lond) 533:773-785.

Ogawa S, Lee TM, Stepnoski R, Chen W, Zhu XH, Ugurbil K (2000) An approach to probe some neural systems interaction by functional MRI at neural time scale down to milliseconds. Proc Natl Acad Sci USA 97:11026-11031.

Oliviero A, Tecchio F, Zappasodi F, Pasqualetti P, Salustri C, Lupoi D, Ercolani M, Romani GL, Rossini PM (2004) Brain sensorimotor hand area functionality in acute stroke: insights from magnetoencephalography. NeuroImage 23:542-550.

Palmer GC, Peeling J, Corbett D, Del Bigio MR, Hudzik TJ (2001) T2-weighted MRi correlates with long-term histopathology, neurology scores, and skilled motor behavior in a rat stroke model. Ann NY Acad Sci 939:283-296.

Paxinos G, Watson C (1998) The rat brain in stereotaxic coordinates, Ed 4. San Diego: Academic.

Pineiro R, Pendlebury S, Johansen-Berg H, Matthews PM (2002) Altered hemodynamic responses in patients after subcortical stroke measured by functional MRI. Stroke 33:103-109.

Ramos-Cabrer P, Weber R, Wiedermann D, Hoehn M (2005) Continuous noninvasive monitoring of transcutaneous blood gases for a stable and persistent BOLD contrast in fMRI studies in the rat. NMR Biomed $18: 440-446$.

Rossini PM, Calautti C, Pauri F, Baron JC (2003) Post-stroke plastic reorganisation in the adult brain. Lancet Neurol 2:493-502.

Rossini PM, Altamura C, Ferretti A, Vernieri F, Zappasodi F, Caulo M, Pizzella V, Del Gratta C, Romani GL, Tecchio F (2004) Does cerebrovascular disease affect the coupling between neuronal activity and local haemodynamics? Brain 127:99-110.

Sakatani K, lizuka H, Young W (1990) Somatosensory evoked potentials in rat cerebral cortex before and after middle cerebral artery occlusion. Stroke 21:124-132.

Sauter A, Reese T, Porszasz R, Baumann D, Rausch M, Rudin M (2002) Recovery of function in cytoprotected cerebral cortex in rat stroke model assessed by functional MRI. Magn Reson Med 47:759-765.

Schallert T, Woodlee M, Fleming SM (2002) Disentangling multiple types of recovery from brain injury. In: Pharmacology of cerebral ischemia (Krieglstein J, Klumpp S, eds), pp 201-216. Stuttgart, Germany: Medpharm.

Schmitz B, Bock C, Hoehn-Berlage M, Kerskens CM, Bottiger BW, Hossmann KA (1998) Recovery of the rodent brain after cardiac arrest: a functional MRI study [Erratum (1998) 40:340]. Magn Reson Med 39:783-788.

Silverman J, Muir III WW (1993) A review of laboratory animal anesthesia with chloral hydrate and chloralose. Lab Anim Sci 43:210-216.

Smirnakis SM, Schmidt MC, Weber B, Tolias AS, Augath M, Logothetis NK (2007) Spatial specificity of BOLD versus cerebral blood volume fMRI for mapping cortical organization. J Cereb Blood Flow Metab 27:1248-1261.

Strupp JP (1996) Stimulate: a GUI based fMRI analysis software package. NeuroImage 3:S607.

Ueki M, Linn F, Hossmann KA (1988) Functional activation of cerebral blood flow and metabolism before and after global ischemia of rat brain. J Cereb Blood Flow Metab 8:486-494.

Waltz AG (1969) Red venous blood: occurrence and significance in ischemic and nonischemic cerebral cortex. J Neurosurg 31:141-148.

Weber R, Ramos-Cabrer P, Wiedermann D, van Camp N, Hoehn M (2006) A fully noninvasive and robust experimental protocol for longitudinal fMRI studies in the rat. NeuroImage 29:1303-1310.

Wegener S, Weber R, Ramos-Cabrer P, Uhlenkueken U, Sprenger C, Wiedermann D, Villringer A, Hoehn M (2006) Temporal profile of T2weighted MRI distinguishes between pannecrosis and selective neuronal death after transient focal cerebral ischemia in the rat. J Cereb Blood Flow Metab 26:38-47.

Wikström H, Roine RO, Aronen HJ, Salonen O, Sinkkonen J, Ilmoniemi RJ, Huttunen J (2000) Specific changes in somatosensory evoked magnetic fields during recovery from sensorimotor stroke. Ann Neurol 47:353-360. 